\title{
Functional paraganglioma with tumor thrombus in the inferior vena cava, first case report
}

\author{
Xiaoqiang Xue ${ }^{1}$, Dong Wang ${ }^{1}$, Yu Xiao' ${ }^{2}$ Zhigang Ji ${ }^{1}$, Yi Xie ${ }^{1}$ \\ ${ }^{1}$ Department of Urology, Peking Union Medical College Hospital, Chinese Academy of Medical Sciences and Peking Union Medical College, \\ Beijing, China; ${ }^{2}$ Department of Pathology, Peking Union Medical College Hospital, Chinese Academy of Medical Sciences and Peking Union \\ Medical College, Beijing, China \\ Correspondence to: Yi Xie; Zhigang Ji. Peking Union Medical College Hospital, Chinese Academy of Medical Sciences and Peking Union Medical \\ College, Beijing 100730, China. Email: xieyi6262@163.com; jizhiganguro@126.com.
}

\begin{abstract}
Pheochromocytoma (PHEO) is a rare neuroendocrine that tumor originated from the adrenal medulla that secrets catecholamines. Tumors from extra-adrenal chromaffin tissues are called extra-adrenal PHEO or paraganglioma (PGL). To our knowledge, adrenal PHEO and subclinical PGL with inferior vena cava (IVC) invasion had been sporadically reported, while functional PGL with IVC tumor thrombus has not been publicly reported yet. Perioperative management of those diseases is less well established because of their multidisciplinary nature and rarity. We herein present a case of primary malignant PGL with IVC invasion. A 16-year-old female patient with a history of severe paroxysmal hypertension was admitted to Peking Union Medical College Hospital on suspicion of retroperitoneal mass. In-house diagnostic work-up revealed a malignant PGL with IVC invasion, inferior mesenteric artery encasement and, aorta engagement. Multi-disciplinary discussions were held and careful preoperative preparation plans were made. After everything was ready, the functional PGL and tumor thrombus were completely resected, then a reconstruction of IVC was performed. The patient was discharged on postoperative day 14 and all her clinical symptoms disappeared afterward. No evidence of tumor residual or metastasis was found in the subsequent six months of follow-up. Gene tests were made for her and her family. Albeit its rarity, functional PGL with IVC invasion is not unresectable, a multi-disciplinary task force should be established to settle down every detail. We recommended 3-dimensional imaging reconstruction for gaining a better anatomic understanding. Literature reviews showed that complete resection is the premise of a good prognosis. In particular cases, complementary or alternative therapy like chemotherapy and ${ }^{131} \mathrm{I}$-metaiodobenzylguanidine might help, family hereditary genetic tests are advised as well.
\end{abstract}

Keywords: Paraganglioma (PGL); pheochromocytoma (PHEO); inferior vena cava tumor thrombus (IVC tumor thrombus); first case report

Submitted Jan 19, 2021. Accepted for publication Mar 04, 2021.

doi: $10.21037 / \mathrm{tau}-21-50$

View this article at: http://dx.doi.org/10.21037/tau-21-50

\section{Introduction}

Pheochromocytoma (PHEO) and paraganglioma (PGL) are rare neuroendocrine tumors that differ mainly in location, PHEOs are adrenal, while PGLs could be found alongside the length of the sympathetic cell chain, starting from the neck to the pelvic. It is most commonly found in the retroperitoneal sites, corresponding anatomical part of
Zuckerkandl's organ. Typical symptoms of PHEO and PGL including the noticeable triad of palpitations, headaches, and sweating, nevertheless, cases of asymptomatic PHEO and PGL are increasing owing to the prevalence of physical examination, family and germline mutation testing $(1,2)$. Unlike cases of PHEO with inferior vena cava (IVC) tumor thrombus that had been sporadically reported (3-5), PGL with IVC tumor thrombus is even rarer and only two cases 
had been publicly reported before, both of them were subclinical, asymptomatic, and accidentally discovered $(6,7)$, report of PGL with the neuroendocrine function that invades the IVC is lacking. We present the following article in accordance with the CARE reporting checklist (available at http://dx.doi.org/10.21037/tau-21-50).

\section{Case presentation}

A 16-year-old female patient with a history of severe paroxysmal hypertension was admitted to Peking Union Medical College Hospital in June 2020. Her recorded highest blood pressure (BP) was 190/120 mmHg, accompanying symptoms included palpitation and sweating. She had visited two large tertiary hospitals since September 2019 while no surgeon there was willing to do the operation. In the aspect of etiologic diagnosis, the out-patient test of plasma metanephrine (MN) and normetanephrine $(\mathrm{NMN})$ were $0.06 \mathrm{nmo1} / \mathrm{L}$ (reference range $<0.5 \mathrm{nmol} / \mathrm{L}$ ) and $21.24 \mathrm{nmo} 1 / \mathrm{L}$ (reference range $<0.9 \mathrm{nmol} / \mathrm{L}$ ), respectively, 24-h urinary norepinephrine was $899.44 \mu \mathrm{g} / 24 \mathrm{~h}$ (reference range, 16.69-40.65 $\mu \mathrm{g} / 24 \mathrm{~h}$ ), showing her high BP should be a result of catecholamine-induced vasoconstriction. In the aspect of localizing diagnosis, positron emission tomography/computed tomography (PET/CT) scanning indicated an irregular soft tissue mass was found in the right front of the abdominal aorta (AA), its maximum standard uptake value (SUVmax) was 20.7, and a soft tissue within the IVC with a maximum SUV of 16.0, multiple rounded lymph nodes around the bifurcation of AA could be detected as well, which should be considered for metastasis. Further ${ }^{131}$ I-metaiodobenzylguanidine (MIBG) fusion imaging suggested it should be consistent with PGL. A diagnostic biopsy guided by ultrasound proved the above diagnosis.

A preoperative computed tomography urography (CTU) was conducted on $16^{\text {th }}$ June 2020 to depict the anatomy radiologically (Figure 1). Then a 3-dimensional imaging reconstruction was made by a related technology company using her CTU films (Figure 2).

A multi-disciplinary task force led by urologists was established, joined by endocrinologists, anesthesiologists, vascular surgeons, radiologists, intensive care unit (ICU) physicians, scrub nurses. The patient was initiated on 10-mg phenoxybenzamine hydrochloride, three times a day, and 30-mg of nifedipine daily to control her BP, another 5 -mg of bisoprolol fumarate was taken daily to slow down heart rates (HR). Intravenous hydration was adopted as well in case of postoperative hypotension. Medications were adjusted dynamically according to the patient's clinical symptoms like dizziness, palpitation, and postural hypotension. After a month's drug preparation, her final prescription was: $20-\mathrm{mg}$ phenoxybenzamine hydrochloride, three times a day, plus 25-mg metoprolol, twice a day. Under this circumstance her supine BP was around 110 $120 / 70-80 \mathrm{mmHg}, \mathrm{HR}$ at $90-100$ beats per minute (bpm) while standing BP was $80-100 / 50-60 \mathrm{mmHg}, \mathrm{HR}$ at 110 $120 \mathrm{bpm}$, she had gained 10 kilogram's weight in the last 3 months.

The operation day was $13^{\text {th }}$ July 2020 , to protect the right ureter, a double-J stent was placed preoperatively. A longitudinal incision was made in the middle of the abdomen, starting from just below the xiphoid process to $2 \mathrm{~cm}$ above the upper edge of the pubic symphysis. An enlarged lymph node that was about $1 \mathrm{~cm}$ in diameter was found at the bifurcation of AA, no clear metastasis was found elsewhere. Intraoperative exploration showed the tumor, about $6 \mathrm{~cm}$ in length, encased the inferior mesenteric artery (IMA) and adhered tightly to the AA (Figure 2). Right approach exploration indicated the tumor invaded the IVC and could not be separated. Therefore, we decided to lift the tumor from the AA by ligating and disconnecting the IMA. Then the proximal and distal ends of the IVC were dissociated and controlled by auricular forceps. The anterior wall of the IVC was incised alongside the tumor. The tumor, local IVC wall, and tumor thrombus were removed as a whole, the residual wall of the IVC was patched by artificial blood vessel graft. Two abdominal drainage tubes were placed, and the incision was sutured layer by layer. The operation lasted for about $7 \mathrm{~h}$, the patient's BP fluctuated up to $190 / 110 \mathrm{mmHg}$ during the operation, IVC blocking time was 49 minutes. Intraoperative blood loss was about $2,500 \mathrm{~mL}$, intraoperative red blood cell transfusion was $18,00 \mathrm{~mL}$ and plasma transfusion was $400 \mathrm{~mL}$. The patient was transferred to ICU for a transitional treatment and back to the urosurgery ward on postoperative day (POD) 2 , following treatment included heparin anticoagulating and antibiotics, she started oral intake and early ambulation on POD 3, and abdominal drainage tubes were removed on POD 9, her wound stitches were removed on POD 13 and was discharged on POD 14. The pathological result indicated the tumor and part of the IVC wall as a whole with a maximum length of $7.8 \mathrm{~cm}$, a nodular mass could be seen at one end of the vein wall, the size of which was $6.8 \mathrm{~cm}$ $\times 7.5 \mathrm{~cm} \times 3.5 \mathrm{~cm}$. Metastasis was found in the swelling lymph node. The pathological result was accorded with 

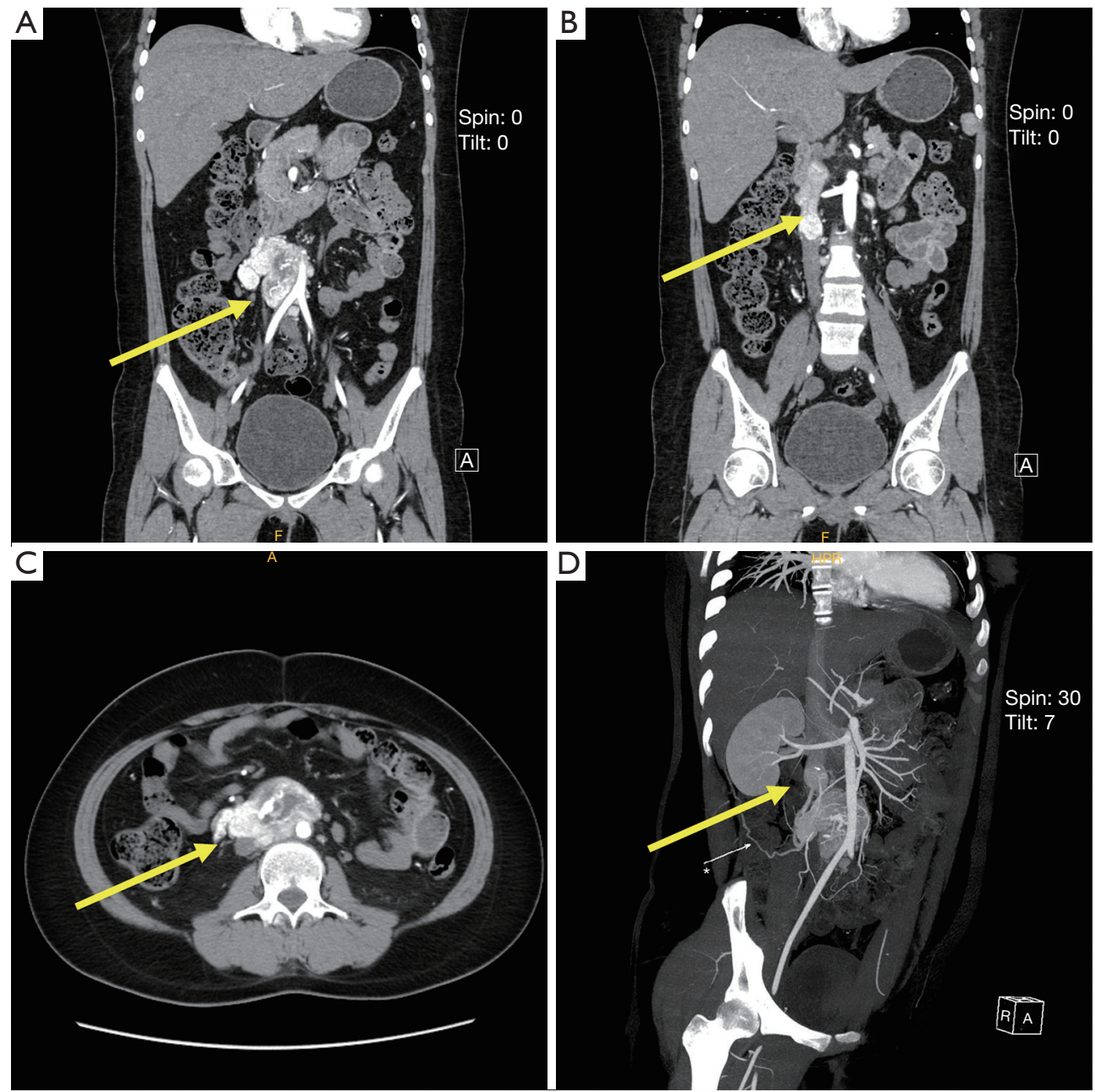

Figure 1 Coronal, axial, and spined view of the tumor in CTU. (A) CTU imaging showed an irregular soft tissue density mass was on the right side of the aorta, from the lower pole of both kidneys to the level of bifurcation of the iliac artery (yellow arrow); (B) IVC tumor thrombus (yellow arrow), its density and enhancement were similar to those of tumor; (C) tortuous blood vessels in the tumor, the tumor was supplied by small branches of the abdominal aorta (yellow arrow); (D) IVC tumor thrombus from a right-anterior view (yellow arrow), tumor blood supply. CTU, computed tomography urography; IVC, inferior vena cava.

PGL. Immunohistochemical results: Melan-A (-), AE1/AE3 (-), CgA (+), Ki-67 (index 3\%), S-100 (+), $\alpha$-inhibin (partial $+)$. The final grading system for adrenal phaeochromocytoma and PGL score (GAPP score) was 3, indicating the tumor was of moderately differentiated type (8).

She had her double-J stent removed and familial gene tested on POD 30. After a 6-month follow-up, no evidence of recurrence was noted (Figure 3), and she reported no complications. Sanger sequencing confirmed that a mutation of exon 4 of succinate dehydrogenase complex subunit $\mathrm{B}(\mathrm{SDHB})$ gene existed in her tumor tissue and peripheral blood, the mutation was c. $423+1 \mathrm{G}>\mathrm{A}$ at chromosome 1:17028599. High-throughput sequencing showed a heterozygous mutation in the peripheral blood of her father, elder brother, and nephew (son of elder brother). No mutation was found in her mother and niece (daughter of elder brother).

This study was approved by the ethics committee of Peking Union Medical College Hospital. All procedures performed in studies involving human participants were in accordance with the ethical standards of the institutional and/or national research committee(s) and with the Helsinki 

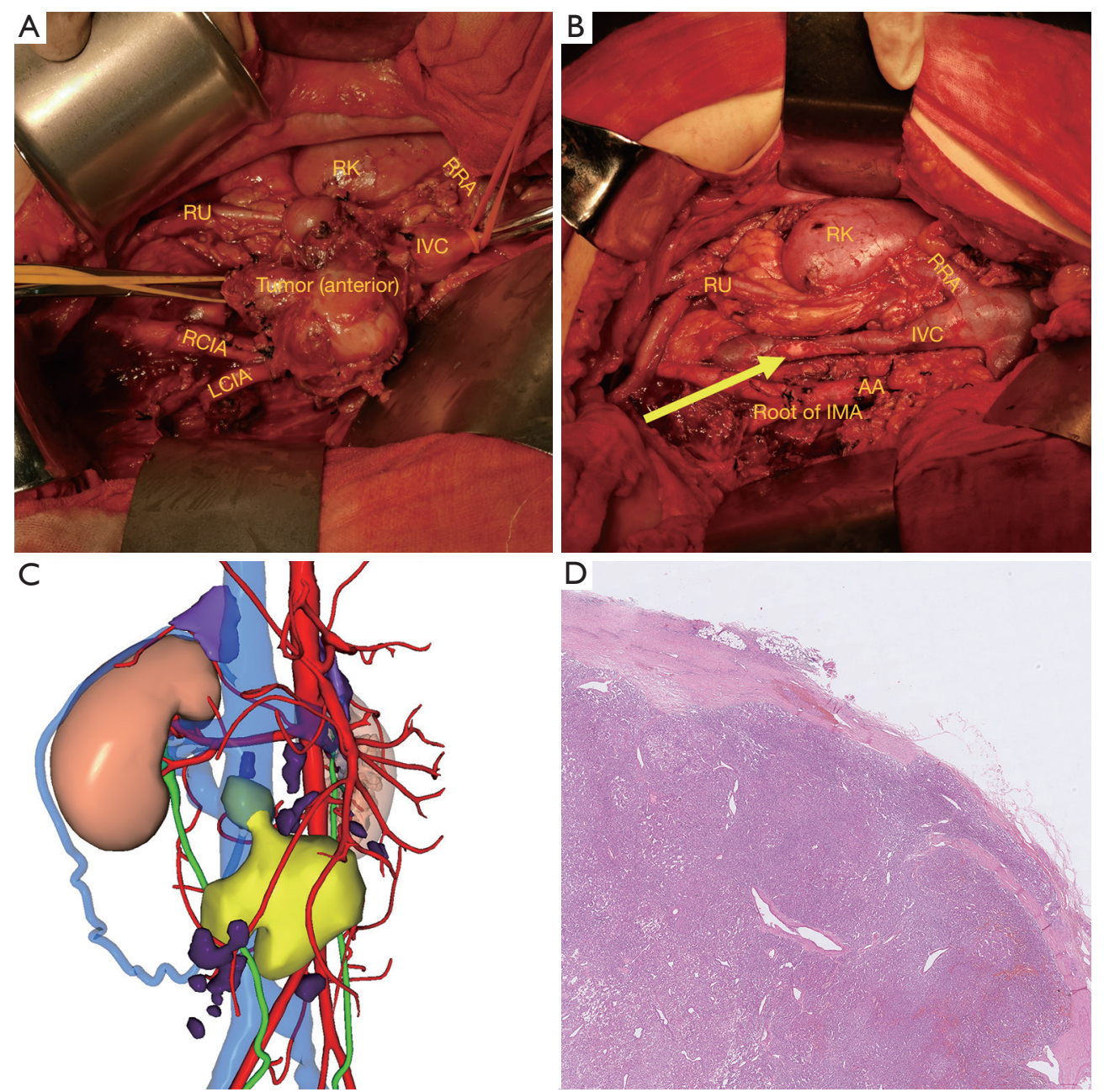

Figure 2 3D reconstruction, intraoperative views, and pathology result. (A) Before resection; (B) after resection and IVC repair (yellow arrow); (C) 3D reconstruction of the tumor, its surrounding vessels and IVC thrombus (yellow: the tumor and IVC tumor thrombus; red: artery; blue: vein; light brown: kidney; violet: lymph nodes; green: ureters); (D) pathological result showing paraganglioma cells with vessel wall invasion. hematoxylin-eosin staining, $\times 100$ magnification equivalent. RCIA, right common iliac artery; LCIA, left common iliac artery; RU, right ureter; RK, right kidney; RRA, right renal artery; IMA, inferior mesenteric artery; AA, abdominal aorta; IVC, inferior vena cava.

Declaration (as revised in 2013). Written informed consent was obtained from the patient and her parents.

\section{Discussion}

PGL is a rare neuroendocrine tumor that constitutes only about $10-20 \%$ of all tumors that secret catecholamine, its typical clinical manifestation includes paroxysmal or persistent hypertension, which can be accompanied by headache, palpitation, and sweating (1). To our knowledge, this is the first case of functional PGL with IVC tumor thrombus reported, all reported PGL cases with IVC tumor thrombus and their characteristics are listed in Table 1. The malignancy of PGL could only be told when local invasion or distant metastasis happens, which may be found either at the time of diagnosis or years after. Up to 50\% PGLs are estimated to have malignancy compared with its intraadrenal kind (7).

Preoperative CTU imaging is of great significance because it could not only help surgeons with a better understanding of the tumor's general characteristics but also the relationship between the tumor and its adjacent blood vessels as well as organs, therefore, achieving a better understanding of surgical approaches and reducing 

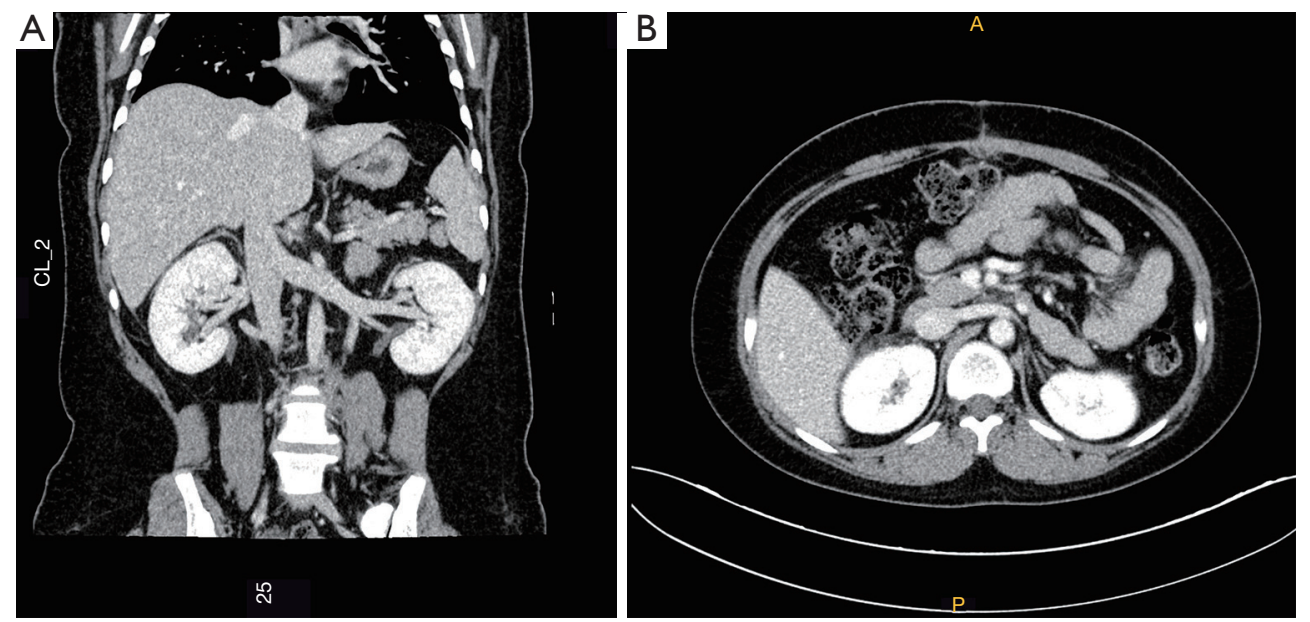

Figure 3 Postoperative follow-up radiology: enhanced CT and CTV of the abdomen. The inferior vena cava was unobstructed, no clear filling defect had been found, the lumen of the inferior vena cava in the lower renal segment was slightly thinner. No local recurrence was found. CT, computed tomography; CTV, CT venography.

Table 1 Comparison of three available cases of paraganglioma with IVC invasion

\begin{tabular}{|c|c|c|c|}
\hline Event & Dossett et al. (7) & Sadamori et al. (6) & This study \\
\hline Sex & Male & Male & Female \\
\hline Age & 46 years & 57 years & 16 years \\
\hline Tumor size & $4.6 \mathrm{~cm}$ in greatest diameter & $7.0 \mathrm{~cm} \times 5.6 \mathrm{~cm}$ & $7.8 \mathrm{~cm} \times 6.5 \mathrm{~cm}$ \\
\hline Positive laboratory tests & Urine NMN and NE & All within normal range & Plasma NMN, urine NE \\
\hline Symptoms & None & None & Hypertension, palpitation, sweating \\
\hline Preoperative preparation & $\alpha-A R B$, intravenous hydration & None & $\alpha-A R B, \beta-A R B$, intravenous hydration \\
\hline Immunohistochemistry & $\begin{array}{l}\mathrm{CgA}(+), \mathrm{S}-100(+), \mathrm{Ki}-67 \\
\text { (index 2-3\%) }\end{array}$ & CgA (+), S-100 (+), Syn (+) & $\begin{array}{l}\mathrm{CgA}(+), \mathrm{S}-100(+), \alpha \text {-inhibin } \\
(\text { partial +), Ki-67 (index 3\%) }\end{array}$ \\
\hline Follow-up & Not mentioned & 12 months of no recurrence & 6 months of no recurrence \\
\hline Gene mutation & $\begin{array}{l}\text { MEN2 (-), VHL (-), SDH series } \\
\text { unknown }\end{array}$ & Not mentioned & $\operatorname{SDHB}(+):$ c. $423+1 \mathrm{G}>\mathrm{A}$ \\
\hline Family pedigree & Not mentioned & Not mentioned & $\begin{array}{l}\text { Heterozygous mutation in her father, } \\
\text { elder brother and nephew }\end{array}$ \\
\hline \multicolumn{4}{|c|}{$\begin{array}{l}\text { CT, computed tomography; MRI, magnetic resonance imaging; CTA, computed tomography angiography; }{ }^{131} \mathrm{I}-\mathrm{MIBG} \text {, } \\
{ }^{131} \mathrm{I} \text {-metaiodobenzylguanidine; PET/CT, positron emission tomography/computed tomography; NMN, normetanephrine; NE, } \\
\text { norepinephrine; } \alpha \text {-ARB, } \alpha \text {-adrenergic receptor blocker; } \beta \text {-ARB, } \beta \text {-adrenergic receptor blocker; IVC, inferior vena cava; AA, abdominal aorta; } \\
\text { RCIA, right common iliac artery; LCIA, left common iliac artery; IMA, inferior mesenteric artery; MEN2, multiple endocrine neoplasia type 2; } \\
\text { VHL, von Hippel-Lindau; SDHB, succinate dehydrogenase complex subunit b. }\end{array}$} \\
\hline
\end{tabular}


unnecessary surgical exploration. MIBG scanning offers a good specificity of $95-100 \%$ while suffers from a relatively imperfect sensitivity of $85 \%$, as a result, patients with negative MIBG results could not exclude the possibility of PGL (9).

Radical resection is the definitive treatment for retroperitoneal PGL, a retrospective study showed the 5 -year disease-free survival rate could benefit significantly from surgical resection (19\% vs. $75 \%$ ) (10). Although tumor size or functional status was not a predictor of survival, the operation procedure could be risky because any stimulation of the functional tumor might result in an impulsive release of catecholamines and resultant hypertension crisis (10-12). Tumors in the Zuckerkandl's organ region that encase or engage great vessels would be a larger conundrum. Note that operations on the other two PGL cases (Table 1) seemed to be more aggressive because the resection involved more organs and vessels, we reckoned this was partly because those tumors were nonfunctional, which could lead to an insidious tumor growth without any clinical symptom and late discovery. A good preoperative preparation is the premise of a safe operation. In this case, we used a computer program to build a three-dimensional reconstruction of the tumor, blood vessels, and organs, which could be checked, rotated, and zoomed in and out on our smartphones and tablets. It might be an extra supplement to traditional CT scanning. At the same time, no matter clinical or subclinical, proper medication preparation using $\alpha$-adrenergic receptor blocker plus intravenous hydration are recommended in case of postoperative vasodilation and the consequent hypotension. A multi-disciplinary task force should be built for better communications. Endocrinologists, vascular surgeons, anesthesiologists, radiologists, ICU doctors and scrub nurses are must-invited while general surgeons and doctors of other disciplines are optional regarding specific situations. Once well prepared, IVC or other great vessel invasion is not a contraindication of surgical resection.

In the aspect of surgical techniques, unlike the adrenocortical carcinoma and renal cell carcinoma (RCC) that invade the IVC, operative protocols of adrenomedullary tumor and PGL-related tumor thrombus were seldom presented owing to their rarity. As a result, experience in dealing with RCC and malignant adrenal tumors with IVC invasion may be referred. Considering the relationship of the primary tumor, thrombus, and its neighboring vessels, surgical approaches and procedures could be different. Upon the surgical maneuver on radical nephrectomy and thrombectomy, protecting the renal function of the contralateral kidney is of priority. Thereafter, orders of clamping and releasing veins should be strictly kept during the vessel occlusion procedure, namely, clamping from caudal IVC to the opposite renal vein to the cephalic IVC, while releasing from the cephalic IVC to the renal vein to the caudal IVC, regarding the sites of the occlusion, affiliated branches of the IVC (lumbar vein, gonadal vein, etc.) may also need ligation (13). In cases involving the hepatic veins or above, the occlusion would be more complex (14). On the other hand, as the location of the thrombus is commonly higher, adrenal cancer with IVC invasion may require more extensive surgical procedures, like cardiopulmonary bypass or hypothermic circulatory arrest, controlled hypotension, and ipsilateral nephrectomy, concerning the tumor size and thrombus protrusion extent (15). Apropos this case, preoperative radiology showed that the tumor and its thrombus were below the level of bilateral renal veins, hence renal function protecting, and the above-mentioned orders were less emphasized, on the contrary, the communicating vein between the tumor and right kidney should be brought to the forefront. The top operative difficulty, in this case, was the ligation of the hidden communicating vessels and the IMA to the tumor, for it straddled the AA and nearly the bifurcation of the iliac artery, any mis-operation would cause arterial hemorrhage. After the removal of the primary tumor, a clear view of the surgical field would be present for the following thrombectomy.

The tumor thrombus is normally an irregular and pliable mass, the floating ones might be completely pulled out as a whole. For those with adhesion to the IVC wall, segmental resection and repairment are needed. The IVC wall is usually repaired with 5-0 polypropylene nonabsorbable suture. Graft repairment or artificial vessel replacement should be performed together with vascular surgeons in suspected after-suturing angio-stenosis cases. In this case, an artificial vessel graft was used.

Some studies showed chemotherapy might be a complement or alternative in particular cases. Regimens of cyclophosphamide, vincristine, and dacarbazine usually took effect after 2-4 courses of treatment, its complete response rate, partial response rate, and stable disease rate was $4 \%$, $37 \%$ and $14 \%$, respectively (16). However, the duration of response lasted for 1.6 to 3.3 years, and thereafter a survival benefit was not significant compared with earlier studies $(10,16)$. Treatment with ${ }^{131} \mathrm{I}-\mathrm{MIBG}$ is only effective for patients with positive MIBG radionuclide imaging, the stable disease or partial response rate was more than $50 \%$, 
5 -year survival after original diagnosis was $68 \%$, its most common side effect were myelosuppression, myelodysplastic syndrome, and myeloid leukemia (17-19).

Familial gene tests should be emphasized, to date, there have been at least 17 pathogenic genes that are thought to be relevant with PGL, including RET, von Hippel-Lindau (VHL), succinate dehydrogenase complex series (SDHA, SDHB, etc.), and neurofibromatosis type 1 (NF1). Amongst them, SDHB gene mutation accounts for about $10 \%$ of the total mutation and more than $40 \%$ of the total malignant PGL is related to it (20). Current studies suggested that SDHB gene mutation was highly correlated with PGL's early onset, recurrence, and malignancy, and the penetrance of familial SDHB gene mutation carriers increased with age $(21,22)$. In this case, although the 6-month follow-up result was pleasing, lifelong follow-up is suggested for the patient because of her identity as a familial SDHB gene carrier that PGL might recur or metastasize later, so are her father, brother, and nephew.

Despite its strengths, our study is not lacking in limitations. That is, the rarity nature because it is very difficult to meet such patients. Moreover, long-term outcomes of these cases are to be seen or to be summarized by expanding the cases and the follow-up time.

\section{Conclusions}

Albeit its rarity and complexity, functional PGL with IVC invasion should not be regarded as a surgical contraindication. Medication preparation, threedimensional reconstruction, and multi-disciplinary discussion could help to contribute a higher possibility of surgery. Surgical resection is the premise of a better prognosis, chemotherapy, and radiotherapy are novel. Genetic tests are recommended to all patients and their families.

\section{Acknowledgments}

Funding: None.

\section{Footnote}

Reporting Checklist: The authors have completed the CARE reporting checklist. Available at http://dx.doi.org/10.21037/ tau-21-50

Conflicts of Interest: All authors have completed the ICMJE uniform disclosure form (available at http://dx.doi. org/10.21037/tau-21-50). The authors have no conflicts of interest to declare.

Ethical Statement: The authors are accountable for all aspects of the work in ensuring that questions related to the accuracy or integrity of any part of the work are appropriately investigated and resolved. This study was approved by the ethics committee of Peking Union Medical College Hospital. All procedures performed in studies involving human participants were in accordance with the ethical standards of the institutional and/or national research committee(s) and with the Helsinki Declaration (as revised in 2013). Written informed consent was obtained from the patient and her parents.

Open Access Statement: This is an Open Access article distributed in accordance with the Creative Commons Attribution-NonCommercial-NoDerivs 4.0 International License (CC BY-NC-ND 4.0), which permits the noncommercial replication and distribution of the article with the strict proviso that no changes or edits are made and the original work is properly cited (including links to both the formal publication through the relevant DOI and the license). See: https://creativecommons.org/licenses/by-nc-nd/4.0/.

\section{References}

1. Neumann HPH, Young WF Jr, Eng C. Pheochromocytoma and Paraganglioma. N Engl J Med 2019;381:552-65.

2. Lucon AM, Falci R Jr, Praxedes JN, et al. Multicentric pheochromocytoma and involvement of the inferior vena cava. Sao Paulo Med J 2001;119:86-8.

3. Shigemura K, Tanaka K, Arakawa S, et al. Malignant pheochromocytoma with IVC thrombus. Int Urol Nephrol 2007;39:103-6.

4. Qureshi SS, Medhi SS. Large adrenal ganglioneuroma with left inferior vena cava: implications for surgery. Pediatr Surg Int 2008;24:455-7.

5. Dunn CW, Snyder WH, Ring WS, et al. Pheochromocytoma with extension into the inferior vena cava: a case report. Surgery 1992;111:472-4.

6. Sadamori H, Monden K, Hioki M, et al. Extra-Adrenal Retroperitoneal Paraganglioma with Extensive Duodenal Invasion and Inferior Vena Cava Tumor Thrombus. J Gastrointest Surg 2016;20:1532-4.

7. Dossett LA, Rudzinski ER, Blevins LS, et al. Malignant 
pheochromocytoma of the organ of Zuckerkandl requiring aortic and vena caval reconstruction. Endocr Pract 2007;13:493-7.

8. Kimura N, Takayanagi R, Takizawa N, et al. Pathological grading for predicting metastasis in phaeochromocytoma and paraganglioma. Endocr Relat Cancer 2014;21:405-14.

9. Lee KY, Oh YW, Noh HJ, et al. Extraadrenal paragangliomas of the body: imaging features. AJR Am J Roentgenol 2006;187:492-504.

10. Sclafani LM, Woodruff JM, Brennan MF. Extraadrenal retroperitoneal paragangliomas: natural history and response to treatment. Surgery 1990;108:1124-9; discussion 1129-30.

11. Chen J, Liu C, Liu C, et al. Anesthetic management of gigantic pheochromocytoma resection with inferior vena cava and right atrium tumor thrombosis: a case report. BMC Anesthesiol 2019;19:71.

12. Kassahun W'T. Update on the optimal management of patients with vascular extension of pheochromocytoma. Vascular 2015;23:297-304.

13. Wang B, Li H, Ma X, et al. Robot-assisted Laparoscopic Inferior Vena Cava Thrombectomy: Different Sides Require Different Techniques. Eur Urol 2016;69:1112-9.

14. Wang B, Li H, Huang Q, et al. Robot-assisted Retrohepatic Inferior Vena Cava Thrombectomy: First or Second Porta Hepatis as an Important Boundary Landmark. Eur Urol 2018;74:512-20.

15. Chiche L, Dousset B, Kieffer E, et al. Adrenocortical carcinoma extending into the inferior vena cava: presentation of a 15 -patient series and review of the

Cite this article as: Xue X, Wang D, Xiao Y, Ji Z, Xie Y. Functional paraganglioma with tumor thrombus in the inferior vena cava, first case report. Transl Androl Urol 2021;10(4):18131820. doi: $10.21037 /$ tau-21-50 literature. Surgery 2006;139:15-27.

16. Niemeijer ND, Alblas G, van Hulsteijn LT, et al. Chemotherapy with cyclophosphamide, vincristine and dacarbazine for malignant paraganglioma and pheochromocytoma: systematic review and meta-analysis. Clin Endocrinol (Oxf) 2014;81:642-51.

17. van Hulsteijn LT, Niemeijer ND, Dekkers OM, et al. (131)I-MIBG therapy for malignant paraganglioma and phaeochromocytoma: systematic review and meta-analysis. Clin Endocrinol (Oxf) 2014;80:487-501.

18. Gonias S, Goldsby R, Matthay KK, et al. Phase II study of high-dose [131I]metaiodobenzylguanidine therapy for patients with metastatic pheochromocytoma and paraganglioma. J Clin Oncol 2009;27:4162-8.

19. Rutherford MA, Rankin AJ, Yates TM, et al. Management of metastatic phaeochromocytoma and paraganglioma: use of iodine-131-meta-iodobenzylguanidine therapy in a tertiary referral centre. QJM 2015;108:361-8.

20. Lenders JW, Duh QY, Eisenhofer G, et al. Pheochromocytoma and paraganglioma: an endocrine society clinical practice guideline. J Clin Endocrinol Metab 2014;99:1915-42.

21. Bausch B, Wellner U, Bausch D, et al. Long-term prognosis of patients with pediatric pheochromocytoma. Endocr Relat Cancer 2013;21:17-25.

22. Jochmanova I, Wolf KI, King KS, et al. SDHB-related pheochromocytoma and paraganglioma penetrance and genotype-phenotype correlations. J Cancer Res Clin Oncol 2017;143:1421-35. 